\title{
DNA Vaccination Target on Amyloid Oligomer also Reduce tau Pathology and Improve Synaptic Function in Aged 3xTg-AD Mice
}

sha sha ( $\nabla$ yanbingxingya@163.com)

China Medical University First Hospital https://orcid.org/0000-0001-8689-4305

xiaona Xing

The Third Affiliated Hospital of Shenzhen University: Shenzhen Luohu Hospital Group Luohu People's Hospital

\section{Tao Wang}

Northeastern University

\section{Ying Li}

China Medical University First Hospital

\section{RongWei Zhang}

China Medical University First Hospital

\section{Xueli Shen}

China Medical University First Hospital

\section{Yunpeng Cao}

China Medical University First Hospital

\section{Le Qu}

China Medical University First Hospital

\section{Research}

Keywords: Immunotherapy, A $\beta$ oligomers,tau phyperphosphorylation,synaptic function,microglia activation

Posted Date: October 19th, 2020

DOI: https://doi.org/10.21203/rs.3.rs-92180/v1

License: (c) (1) This work is licensed under a Creative Commons Attribution 4.0 International License. Read Full License 


\section{Abstract}

Background: Active immunotherapy has been widely used as a potential therapeutic method for both treatment and prevention of Alzheimer's disease (AD). Amyloid deposition and tau hyperphosphorylation are main pathological hallmarks of $A D$. Reduction in both is required to regulate synaptic proteins neurotransmission which can finally protect cognitive function. Growing evidence suggests that the most toxic $\beta$-Amyloid peptide $(A \beta)$ oligomers can be detected from the very beginning of $A D$ development, so it is wise for us to develop effective therapy method focus on $A \beta$ oligomers rather than monomers.

Methods: The 3×Tg-AD mice were randomly divided into two groups immunized with $p(A B 3-10) 10-M T$ and PBS respectively. The PBS group was used for positive group while C57/B6 mice was used for negative group. ELISA was used to detect the antibody titers and Morris Water Maze was to analysis the cognitive function. The $A \beta$, Tau hyperphosphorylation, Neuron and synaptic protein were detected through immunohistochemistry and western blot.

Results: Mice immunized with $\mathrm{p}(\mathrm{AB} 3-10)_{10} \mathrm{MT}$ can not only reduce the levels of $A \beta$ oligomers and plaque deposits but also protect neuron as well as synaptic function, finally prevent the decline spatial memory in transgenic mice.

Conclusions: Our novel DNA genetic vaccine is highly safe and effective, thus providing strong evidence for the treatment and prevention of early $A D$.

\section{Introduction}

Alzheimer's disease (AD), characterized by senile plaques and neurofibrillary tangles that eventually lead to neuronal degeneration, is a major cause of cognitive dysfunction in the elderly[1]. $A \beta$ oligomer, rather than $A \beta$ oligomer, is considered to be the primary initiator of $A D$, which can induce series of pathophysiological changes such as tau hyperphosphorylation, inflammatory response, oxidative stress, synaptic dysfunction, and neurodegeneration[2].Both active and passive immunotherapy can effectively eliminate amyloid deposits and the pathophysiological changes induced by $A \beta$ in human and animal models[3, 4].Otherwise, there was no significant difference between the control group and the treatment group in the interval between progression to severe cognitive impairment[5].Monoclonal antibodies such as solanezumab, gantenerumab, crenezumab, aducanumab have been detected in $\mathrm{MCl}$ (Mild Cognitive Impairment) stage and in patients at high risk of developing $A D[6]$. At present, lack of specific treatment for the most toxic oligomers may be the main defect of $A D$ immunotherapy, because target normal sA $\beta$ can interfere with its crucial physiological functions of $A \beta$, including neuroprotection, modulation synaptic elasticity, memory consolidation and maintenance innate immunity[7].

There was a strong correlation between soluble $A \beta$ and tau in the AD pathocascade which may happen before the occurrence of senile plaques and neurofibrillary tangles. Besides, Tau down-regulation the express of $A \beta$. It was confirmed that anti-A $\beta$ oligomer therapy reduces tau protein-related pathological changes in both animal and clinical trials[8]. 
$A \beta$ oligomer binds to the plasma membrane to open its calcium potential channel to allow its inflow into the cell and down-regulate the activation of phosphokinase in tau phosphorylation. For example, AMPK activates a unique phosphorylation site at ser422 in AD progress.As disease progressed, $A \beta$ is thought to activate Fyn phosphatase, striatal-enriched protein tyrosine phosphatase(STEP), eventually leading to synaptic loss and dendrite collapse[9]. Furthermore,reducing soluble $A \beta$ without disturbing soluble tau can not improve cognitive impairment; therefore, saving cognitive impairment in transgenic mouse requires reduction of both soluble $A \beta$ and tau levels[10]. Soluble $A \beta$ oligomers began to damage synaptic function and affect cognition from the very onset in $A D$ before plaque deposition, so early intervention of the disease is necessary to get the most dramatic benefits. Moreover, synaptic associated proteins take an important role in modulating memory conduction.

Dynamin 1 plays an important role in the release of synaptic vesicles, while PSD-95 regulates the maturation and elasticity of synapses. Both of them can be degragated by calpain activation which is induced by $A \beta$ accumulation[11]. Immune therapy can selective reduce soluble $A \beta$ oligomer, as well as reduce the loss of synaptic-related proteins, which can help further protect spatial memory and synaptic function[12]. The triple-transgenic mouse $(3 \times \mathrm{Tg}-\mathrm{AD}$, harboring APPSwe and tauP301L transgenes on a mutant PS1M146V knock-in background) were representative with both amyloid deposition and tau phosphorylation, exhibited deficits in synaptic plasticity which were more similar to those of AD patients from the pathophysiological point of view[13].

The purpose of this study was to investigate the immune characterization of $3 \times \mathrm{Tg}-\mathrm{AD}$ mice immunized with a recombinant $A B 3-10$ gene vaccine and its efficacy on $A D$ related pathology.Our main observation is the effects of our vaccine as follows: 1) Behavioral abnormality 2) amyloid plaque deposits, 3) the amount of insoluble $A \beta \beta$ oligomers, 4) immunoreactivity to monomeric and oligomeric $A \beta$ of induced antibodies, 5) tau phosphorylation, 6) microglial attraction,7) synaptic related protein, 8) microhemorrhage. Finally, the relationship between tau and $A \beta$ and its effect on synaptic function were discussed, which provided a strong basis for further discussion on the effect of $A \beta$ gene vaccine.

\section{Methods}

\section{Animals}

A group of homozygous $3 \times T$ Tg-AD mice were obtained by purchasing breeding pairs from Jackson Laboratory (USA). C57/B6 mice were from Beijing Vital River Laboratory Animal Technology Co., Ltd., China as wild- type (WT) mice. The mice were grown in a sterile environment in the Laboratory Animal Centre of China Medical University, with light/dark cycle of 12 hours. In addition, experiments were performed according to the guidelines of the Animal Care and Use Committee of the China Medical University.

\section{Peptide synthesis and preparation}


The gene was identified based on the cDNA sequence of a gene 3-10 in GenBank Fragment synthesis :5 'TT-Ecori-Cozak-ATG (initial Bcodon)

(Aß3-10)10-TAG(stop codon)-Notl-Xhol-GG-3', and $10 \times$ Aß3-10, was

cloned of mammalian expression vector by EcoRl/ (pcDNA3.1)

Xhol restricts websites.The recombinant plasmid was verified by Notl/

EcoRI digestion and gel electrophoresis. The correct plasmid sequence is

confirmed by nucleotide sequence analysis (Shanghai GeneCore Bio)technology.Recombinant plasmid was amplified in DH5 receptive cells of E. coli and purified using the E.z.n.TM Fastfilter plasmid - free Maxi kit (OMEGA, USA).

\section{Immunization of Mice by In Vivo Electroporation}

The 3×Tg-AD mice were randomly divided into two groups and immunized with $p(A B 3-10) 10-M T$ vaccine $(n=10)$ and PBS $(n=10)$ respectively. The PBS group was used for positive group while C57/B6 mice was used for negative group. Mice with $p(A B 3-10) 10-M T$ were intramuscular injected into the left leg and immunized with 10 times every 3 weeks. After anaesthetized, a pair of 26 electrode needles was inserted into the muscle $5 \mathrm{~mm}$,covering the DNA injection sites, electrical pulses were transmitted using an electric pulse generator (ECM830, BTX Harvard Apparatus company, USA), Output of $6,75 \mathrm{~V}$ pulses at a rate of one pulse per $200 \mathrm{~ms}$ [14]. Orbital venous blood samples were taken for biochemical examination before the first immunization and 10 days after each immunization.

\section{Immunoreactivity of antisera and response to $A \beta$ polymers}

Humoral immune response was detected by enzyme-linked immunoabsorbent assay(ELISA)[15]. In brief, we use 96-microwell plates coated with GST-A $\beta$ proteins to detect the A $\beta$ peptide in serum samples diluted with PBS at 1:1,000. Microtiter wells were treated with blocking buffer (5.0 \% goat serum, $1 \%$ BSA, and $0.05 \%$ Tween-20 in PBS) and left at room temperature for 2 hours. Standard curve was produced by continuous dilutions of the standard 6E10 antibody (monoclonal anti-A $\beta$ antibody, Covance).For western blot analysis, oligomeric $A \beta$ was prepared as described by Dahlgren et al[16]. The peptide was dissolved in $1 \mathrm{mM}$ hexafluoroisopropanol (Sigma) and removed under vacuum in a Speed Vac(Savant, Holbrook, NY). The remaining peptide was in $5 \mathrm{mM}$ in dimethyl sulfoxide (Sigma) concentration. Free ham F-12 medium(Mediatech, Herndon, VA) was added with phenol red at $100 \mathrm{IM}$ and maintained at $4{ }^{\circ} \mathrm{C}$ for $24 \mathrm{~h}$. The sample dilution buffer(Carlsbad, CA) and 16.5\% Tris-Tricine SDS-PAGE separation for NuPage sample. Western blotting was performed using induced anti-sera and an enhanced chemiluminescence system (Amersham, Arlington Heights, IL) as described previously[17].

\section{Morris Water Maze Test}


As described previously, Morris water maze is conducted in a $150 \mathrm{~cm}$ round pool which was fulled with white milk, $40 \mathrm{~cm}$ in diameter, quiet in the centre with room temperature $22^{\circ} \mathrm{C}[18]$. The water maze test was performed within 2 weeks of immunization.The experiment was carried out in a circular tank $1.25 \mathrm{~m}$ in diameter and $0.4 \mathrm{~m}$ high with a digital acquisition camera for monitoring animal behaviour and a computer program for data analysis (ZH0065, Zhenhua Biological Equipment, China). The mice were trained on the visible platform for two days, then on the hidden platform for two days, and were withdrawn from the platform a day later for the probe test.Each mouse was placed in the water from the same position in each quadrant and reached the platform 60 seconds later.Finally, the escape delay, the number of times in the platform position has been crossed, and the percentage of time spent in the target quadrant are analyzed.

\section{Immunohistochemistry of $A \beta$, Tau,Neuron}

Immunohistochemical analysis and quantitative staining sections were described previously[19].A $\beta$ plaques were detected using a monoclonal anti-A $\beta$ antibody $6 \mathrm{E} 10$. HT7 recognizing epitopes 159-163 $(1,40$, Thermo Scientific, Waltham, MA, USA) detected total tau, AT8 and AT180 recognize phosphorylated tau levels. AT8 identified Ser202/Thr205 site while AT180 identified phosphorylated Thr231 site seperately. Quantification of HT7- ,AT8-positive and AT180-positive neurons were quantified and the number of pixels (A.U.) represents total tau (HT7) or hyperphosphorylated tau (AT8 and AT180) load.Both cortex and hippocampus were performed of $A \beta$ plaques and tau protein. In order to better identify neurodegenerative sensitive neurons, neuron-specific nuclear antigen NeuN antibody and pPKR antibody were used as markers. To be representative and accurate, images were analyzed using Image J(developed by W.S. Rasband, National Institutes of Health, Bethesda MD, USA, 1.47g) to obtain protein loads (percentage of staining rea).pPKR was performed on thirty cortical gray matter fields magnification $\times 20$ from each brain region to determine the number of NeuN-positive neurons in each field.

\section{Prussian blue for cerebral hemorrhage}

To stain the microhemorrhage, series of coronal sections of the brains of laboratory and control mice were mounted on gelatin coated slides and stained with Prussian blue working fluid as described earlier [20]. Briefly, parts of the brain were incubated with the same amount of mixture distilled $\mathrm{H}_{2} \mathrm{O}$ potassium ferrocyanide and $20 \%$ hydrochloric acid for 30 minutes. The subsequent sections are washed with water, redyed with a quick 10 minute red solution, rewashed with water, dehydrated and covered with glass slides using Depex mounting media (BDH Laboratory Supplies, England).

\section{Immunofluorescence to detect relationship between microglial and $A \beta$}

For double immunofluorescence staining, the frozen sections were placed in fetal bovine serum(1:20) and incubated at room temperature for $1 \mathrm{~h}$.Sections were incubated overnight at room temperature with monoclonal anti-A $\beta$ antibody $6 \mathrm{E} 10$ (dilution, 1:1000) and rabbit anti Iba-1(1:500). The mixture of goat anti-mouse sections were incubated for $2 \mathrm{~h}$ at room temperature in a mixture of fluorescein isothiocyanate-conjugated goat anti-mouse IgG and Texas Red-conjugated goat anti-rabbit IgG after 
rinsed with PBS with fluorescein isothiocyanate for $2 \mathrm{~h}$. Then the sections were rinsed and covered with PBS/glycerol buffer. The fluorescence micrographs were captured and analyzed by confocal laser microscope.

\section{Western Blot}

We used western blot determine the level of $A \beta$ oligomers, tau protein, calpain and five synaptic proteins in the cerebral homogenate from immunized mice.Protein samples were isolated on $12 \%$ SDS-PAGE gel and transferred to a PVDF membrane. In $20 \mathrm{mM}$ Tris- $\mathrm{HCl}$ (pH 7.4) containing $150 \mathrm{mM} \mathrm{NaCl}$ and $0.05 \%$ Tween 20 (TBS-T), 10\% skim milk closed membrane was washed with TBS-T.Then antibody 6E10 (1:1500),Tau5 (mouse tau antibody 1:200, Thermo scientific, Rockford, IL), AT8 (mouse anti-PHF tau antibody 1:200, Thermo scientific, Rockford, IL),AT180 (mouse anti-PHF tau antibody 1:500 Thermo scientific, Rockford, IL), anti-dynamin 1 (C16, 1:2000, Santa Cruz Biotechnology, Inc., Santa Cruz, CA), anti-PSD-95 (1:500, Invitrogen, CA), anti-synaptophysin (L128, 1:500, Bioworld Technology, Inc., MN, USA), anti-synapsin (1:500, Bioworld Technology, Inc.) or anti- $\beta$-actin (Sigma, MO) for $1 \mathrm{~h}$ at $37^{\circ} \mathrm{C}$. Then, the membrane was incubated with HRP-conjugated IgG (GBI, WA) and protein was detected with enhanced chemiluminescence reagents (ECL, Pierce, IL). Density was performed using the Image J Software.We considered the value obtained in the untreated control group to be $100 \%$. The values of each group are expressed as mean $\pm S E(n=10)$.

\section{Statistical analysis}

All statistical parameters were expressed as mean \pm SE. We used SPSS19 to analyze immunological, neuropathological and behavioral results. Single factor analysis was used to test significant variance (ANOVA) for statistics.P $<0.05$ was considered statistically significant different.

\section{Results}

\section{A $\beta$ DNA vaccines construction and confirmation}

The $p(A B 3-10) 10-M T$ new vaccine was successfully constructed and expressed as indicated in Sha.et al[21].

High anti-A $\beta$ antibody levels were elicited and the immunogenicity of it against different conformation of $A \beta$

The level of anti-A $\beta$ antibody in the plasma samples of mice was detected by ELISA. The anti-A $\beta$ antibodies can be detected in $\mathrm{p}(\mathrm{A} \beta 3-10) 10-\mathrm{MT}$ immunized $3 \times \mathrm{Tg}-\mathrm{AD}$ mice while no antibody titer be discovered in the PBS group mice. Peak antibody levels reached $47.05 \pm 2.72 \mu \mathrm{g} / \mathrm{mL}$ in $\mathrm{p}(\mathrm{A} \beta 3-10) 10-\mathrm{MT}$ group after the tenth vaccination and then sustained thereafter. The PBS group mice did not produce detectable anti-A $\beta$ antibodies(Fig. $1 \mathrm{~A}$ ). Of note, the sustained immune response may further assisted in maintaining antibody-mediated clearance of $A \beta$ and provide protection in $3 \times \mathrm{Tg}-\mathrm{AD}$ mice.The antibody 
exhibited obvious higher concentration in our vaccine group other than the PBS group(Fig. 1B, $\mathrm{p}<$ 0.01).When mice were 16 months old, $A \beta$ oligomers extracted from their hippocampus homogenates were analyzed by Western blot using 6E10. A trimers, tetramers, hexamers, nonamers, dodecamers and fibrils are represented by arrows. $A \beta \beta 3-10) 10-M T$ immunized serum binds strongly to $A \beta 42$ oligomers, but minimally to $A \beta 42$ fibrils(Fig. 1C). This showed that $p(A \beta 3-10) 10-M T$ serum antibody recognizes $A \beta$ oligomer depending on $A \beta$ peptide conformation.

\section{The $p(A \beta 3-10) 10-M T$ vaccine dramatically allievated cognitive impairment in aged $3 \times \mathrm{Tg}-\mathrm{AD}$ mice}

We evaluated cognition after the last vaccination in a Morris water maze test using the transgenic mice and wild-type mice. Compared to control $3 \times \mathrm{Tg}-\mathrm{AD}$ mice, the escape latency for $\mathrm{p}(\mathrm{A} \beta 3-10) 10-\mathrm{MT}$ immunized group was significantly reduced at $3-5$ days of training (Fig. $2 A, p<0.01$ ). In the probe test, initial incubation latency in $p(A B 3-10) 10-M T$ group was significantly shortened(Fig. $2 B, p<0.01$ ), and it was easier to cross the correct platform location more often than the control group(Fig. $2 \mathrm{C}, \mathrm{p}<0.01$ ). There was no difference between the performance of the $p(A B 3-10) 10-M T$ group and the wild-type group mice in Morris water maze test (Pख0.05).In summary, these data clearly indicate that early immunotherapy can effectively improve the spatial memory of aged $3 \times \mathrm{Tg}-\mathrm{AD}$ mice.

\section{Our $\mathrm{p}(\mathrm{A} \beta 3-10)_{10}-\mathrm{MT}$ vaccine prevent the plaque deposits through microglia activation}

The amyloid burden were significant $41 \%$ reductions in the cortex and $50 \%$ reduction in the hippocampus for the $\mathrm{p}(\mathrm{AB3}-10)$ 10-MT immunized mice compared with PBS-immunized group (Fig. 3A). Meanwhile, ELISA results of cerebral homogenate showed that the vaccine immunization also markedly decrease the levels of soluble and insoluble $A \beta 42$ peptide by $44.3 \%$ and $36.4 \%$ respectively compared to unimmunized mice(Figure.3B,p < 0.05). Immunofluorescence staining double labeling results showed that, compared with PBS group, there were more Iba-1 positive cells clustered around senile plaques in the brain of $\mathrm{p}(\mathrm{A} \beta 3-10) 10-\mathrm{MT}$ immunized mice(Fig. 3C),confirming the role of microglia in clearing the senile plaque deposition.We also semiquantitatively assessed perivascular amyloid deposition in $\mathrm{p}(\mathrm{A} \beta 3-10)_{10}$-MT vaccinated and PBS group mice. Thio S-positive vascular amyloid showed there was no significant difference(Pख0.01) between $\mathrm{p}(\mathrm{A} \beta 3-10)_{10} \mathrm{MT}$ vaccinated and PBS group mice (data not shown).

\section{The $\mathrm{p}(\mathrm{AB3}-10)$ 10-MT vaccine markedly reduces tau pathology in different type of tau levels}

We used AT8 and AT180 respectively to assess the changes of phosphorylated tau protein level and pathological changes after active immunization. We observed hyperphosphorylated tau protein in a large number of cell bodies in unimmunized $3 \times \mathrm{Tg}-\mathrm{AD}$ mice. After $A \beta$ vaccination, AT8-positive neurons were severely reduced and the AT8 signal was not as strong compared with the PBS immunized group(Fig. 4A). The same trendency can be seen in the AT180 after immunized with $p(A \beta 3-10)_{10^{-}}$ MT(Fig. 4C). Both AT8 and AT180 were both decreased in p(Aß3-10) $10^{-M T}$ immuzied group (Fig. 4B,D,p $<0.01)$.Protein extracted from the right hemisphere was detected by Western blot to detect AT8, AT180 and total tau levels. Both AT8 and AT180 signals were significantly reduced in vaccinated mice compared 
with the control group. The density analysis showed that both levels decreased significantly when normalized to total tau levels, after $A \beta$ vaccination (Fig. $4 \mathrm{E}, \mathrm{p}<0.05$ ).

\section{Our vaccine prevented the loss of synaptic proteins and protect synaptic function}

Synaptic proteins take an important part in memory regulation. It has been reported that accumulation of $A \beta$ can induce the activation of calpain, thereby degrading of dynamin 1, a protein which promotes the release of synaptic vesicle release[22], and post-synaptic density protein PSD-95, which is essential for synapse maturation and plasticity.Compared to C57/B6 group with the same month age, the level of dynamin 1 and PSD-95 in the brain of untreated $3 \times$ Tg-AD mice was significantly reduced while those in the $p(A \beta 3-10)_{10}-M T$ immunized group did not decrease $(p<0.01)$.Similarly, the synaptophysin, synapsin I and GluR1 were also significantly reduced in untreated group compared to the wild-type group while $p(A B 3-10)_{10}-M T$ immunization prevented their protein decline and improved their cognitive performance (Fig. 5A,p $<0.01$ ).Previous results showed that calpain was activated by $\mathrm{Ca}^{2+}$ influx induced by $\mathrm{N}$-methylD-aspartate(NMDA) receptors which promote degradation of dynamin 1 and PSD-95[23], leading to impaired memory function. The graphs show the levels of synaptic associated protein in mice from three group. Data represent the mean \pm SE for each group(Fig. 5B,p<0.01).

\section{Our $A \beta$ vaccine prevents neuron loss in the immunized $3 \times \mathrm{Tg}-\mathrm{AD}$ mice}

NeuN immunohistochemistry was used to detect the density of neuron in the CA3 region to evaluate the loss of neuron in mice before and after immunization. The density of hippocampal CA3 neurons in mice inoculated with $A \beta$ vaccine was significantly higher than that of $3 \times \mathrm{Tg}-A D$ in the control group (Fig. $6 \mathrm{~A}$ ). Stereological counts of NeuN-positive CA3 neurons showed a $46 \%$ loss in $3 \times \mathrm{Tg}-\mathrm{AD}$ mice immunized with PBS while only $11 \%$ of neurons in mice immunized with $p(A \beta 3-10){ }_{10}-M T$ was lost $(p<0.01)$, indicating that the new vaccine had a protective effect on neuron loss in $3 \times \mathrm{Tg}-\mathrm{AD}$ mice(Fig. 6B).

\section{No Microhemorrhage In The Vaccine Immunized Mice}

Prussian blue histological staining was used to label hemoglobin decomposition products containing hemosiderin. As described in our previous study, microhemorrhages were detected in A $\beta 42$ immunized and $p(A B 3-10)_{10}-\mathrm{MT}$ transgenic mice, ranging in size from large, diffuse areas to small, single cell-sized blue profiles. Quantitative detection was conducted by calculating the number of microhemorrhage in each brain section as shown previously[24]. There was no obvious difference between these two group (Fig. 7A,B). Importantly, microhemorrhages were rarely detected in the PBS and $p(A B 3-10) 10-M T$ vaccinated mice.

\section{Discussion}

Active immunotherapy targeting $A \beta$ was widely carried out in the preclinical studies of $A D$ mouse models and clinical trials of human being[25]. However, neither has achieved significant clinical efficacy. The 
main reason may be the lack of the specific methods to specifically target the most toxic components $A \beta$ oligomers, and thay may interfere with their normal physiological functions[26].

It was reported that tau in the hippocampus of $3 x \operatorname{Tg}-A D$ mice decreased with the removal of $A \beta$ plaques[27]. Otherwise, simply reducing soluble $A \beta$ without changing soluble tau levels could not significantly improve cognitive ability, suggesting that reversal of memory impairments requires the joint action of both factors[28].Studies have shown that $A \beta$ eventually inactivates Fyn by activating Fyn phosphatase, the striatal-enriched protein tyrosine phosphatase (STEP), leading to synaptic loss and dendritic spine collapse[29]. In summary, $A \beta$ may be the trigger of $A D$ pathogenesis while tau may be the bullet[30],and these two factors interact in the pathophysiology of AD.

A recent Phase 3 clinical trial using solanezumab (antibody m266) suggest that it is also effective in patients with mild $A D$, indicating that prevention method may be more beneficial than treatment[31].Studies have shown that even high titers of antibodies in 18 month older $3 \times$ Tg-AD mice after active immunotherapy cannot completely reverse the deposition of $A \beta$ and changes in cognitive function[32].Another possibility for the decreased plaque clearance ability in elderly mice was the increased fibrillar composition and multiple factors, such as cross-linking, glycation, recombination and integration, which promoted the stronger plaque, all of which proved that early intervention therapy was more effective in the incomplete plaque formation[33].

Compared with traditional peptide vaccine, DNA vaccine have many advantages such as being relatively safe, cost efficient, and able to maintain a reasonable level of antigen expression within cells. DNA vaccines can be easily manipulated to modify genes and depending on the type of immune response required. However, due to the low efficiency of naked DNA transfection cells, the immunogenicity of DNA vaccine is low. Gene gun, electroporation and application of immune adjuvant can significantly improve the DNA immunogenicity[34]. Our vaccine improves antibody titers primarily through electroporation of the muscle.

A potential problem of immunotherapy was that the removal of insoluble $A \beta$ may lead to an increase in soluble $A \beta$, especially oligomer conformation which may directly damage cognitive function[35]. Our vaccine $p(A \beta 3-10) 10-M T$ immunotherapy reduced not only the aggregation of $A \beta$, but also soluble $A \beta$ oligomers. Comparison with full-length $A \beta 42, A \beta 3-42$ is more resistant to proteolytic degradation and more prone to aggregation to produce toxic oligomers. Therefore, antibodies directed against 3-10 can remove the most toxic fragment without interfering normal biological function of $A \beta 42[36]$. In the current study, $p(A \beta 3-10) 10-M T$ immunotherapy elicit high titers serum antibodies that were more strongly binding to oligomers than to monomer, effectively reducing $A \beta$ peptides deposition and oligomers, which correlates so near with cognitive dysfunction in various kinds of transgenic mice, can eventually mitigate cognitive deficits.

A large number of studies have shown that microglia can phagocytosis and clear senile plaque deposition, thereby alleviating AD pathology[37].Our vaccine significantly increased more and more microglia clustered near amyloid plaques after immunotherapy indicated that immune therapy could 
change the phagocytic function of microglia and help clear plaque clearance[38].Fluorescence immunolabeling show dark-brown; the plaque densely scattered surrounded microglia with increased cytoplasm and longer synapses tended to be activated.

$A \beta$ and tau share the same structure and biophysical, such as a high $\beta$-sheet content, neurotoxicity and partial resistance to proteolytic degradation. Studies have confirmed that the tau phosphorylation site related to AD is AT8 to detect Ser202 and Thr205, while Th231 identified by AT180 is also an important step of tau phosphorylation, which promotes pathological Tau changes[39].Our data showed a significant reduction in tau phosphorylation after removal of amyloid.Immunization can reduce AT8 and AT180, thereby reducing tau associated pathology[40].Therefore, active immunization with $p(A \beta 3-10)_{10^{-}}$ MT vaccine can simultaneously act on $A \beta$ and tau protein, thus producing encouraging therapeutic effects.

Pre-synaptic and post-synaptic proteins, were more strongly associated with cognitive deficits than $A \beta$ plaques. These two important synaptic proteins, Synapsin I and synaptophysin, are mainly involved in the circulation of synaptic vesicles. The major determinant of synaptic plasticity is post-synaptic protein PSD-95. Dynamin 1, may involved in synaptic vesicle release, which participate in A $\beta$-induced synaptic pathology[41].A $\beta$ aggregation further lead to synaptic dysfunction by degrading calpain-mediated dynamin 1 or PSD-95.Our vaccination significantly reduced both $A \beta$ and Calpain activation, effectively preventing the degradation of dynamin 1 and PSD-95.After immunized $3 \times$ Tg-AD mice with $p(A \beta 3-10) 10-$ $M T$, the activation of calpain in the brain was significantly decreased, which may led to the reduction of dynamin 1 and PSD-95 degradation, eventually protected the synaptic function. Studies have shown that immunotherapy can clear degenerated neurons, and mice that received immunizations did not find severely degenerated neurons. The loss of neurons in our vaccine group was significantly reduced and the health of neurons was improved. This suggests that immunized $3 \times \mathrm{Tg}-\mathrm{AD}$ mice can partially protect neuron loss.

In summary,we have comprehensively described the immunogenicity, efficacy, and mechanism of $\mathrm{p}(\mathrm{A} \beta 3-$ 10) 10 -MT vaccine as early immunotherapy model for $3 \times \mathrm{Tg}-\mathrm{AD}$ mice. Our data firstly showed that active immunization with a DNA plasmid coding for an AB3-10 through in vivo electroporation to induce a humoral immune response against $A \beta$ in the $3 \times T g-A D$ mice, which significantly reduced tau as well as $\beta$ amyloid.Our data also showed that the new vaccine cleared soluble $A \beta$ from the brain, including the most toxic oligomers, preventing the degradation of pre-synaptic and post-synaptic related protein as well as improving the cognitive function. In conclusion, our vaccine, which took $A \beta$ oligomers as the special targets, reduced senile plaques and tau phosphorylation while protected synapse function without microhemorrhages, can be considered to be effective and safe AD candidate vaccine of preventive treatment or early immunotherapy.

\section{Conclusion}


Mice immunized with $p(A \beta 3-10)_{10}-\mathrm{MT}$ can not only reduce the levels of $A \beta$ oligomers and plaque deposits but also protect neuron as well as synaptic function, finally prevent the decline spatial memory in transgenic mice. Our novel DNA genetic vaccine is highly safe and effective, thus providing strong evidence for the treatment and prevention of early $A D$.

\section{Abbreviations}

\section{$A \beta$}

Amyloid-beta; AD:Alzheimer's disease; MT:Melatonin; ANOVA:Analysis of variance; H\&E:Hematoxylin and eosin; PBS:Phosphate saline buffer; WT:Wild-type; PSD-95:postsynaptic density 95; STEP:striatalenriched protein tyrosine phosphatase; MCI:Mild Cognitive Impairment; AMPK:Adenosine Monophosphate Activated Protein Kinase; pPKR:phosphorylated protein Kinase RNA; PVDF:Polyvinylidene Fluoride;

\section{Declarations}

\section{Authors' contributions}

Yunpeng Cao and Sha Sha conceived and designed the experiments. Formal analysis: Xiaona Xing and Sha Sha. Funding acquisition: Yunpeng Cao and Le Qu. Writing: Sha Sha and Le Qu. The author(s) read and approved the final manuscript.

\section{Acknowledgements}

The authors would like to thank Dr. Wang at College of Life and Health Sciences, Northeastern University and Dr. Qu at the department of Dermatology, the First Affiliated Hospital of China Medical University for their support to provide platform and technology support.

\section{Funding}

This work was supported by the National Nature Science Foundation of China of professor Cao[grant number 81371227] and Natural Science Foundation of Liaoning Province of China of Dr. Xing [No.2019MS-200]

\section{Availability of data and materials}

The data used during the current study are available from the corresponding author on reasonable request.

\section{Ethics approval and consent to participate}

All animal studies were approved by the Institutional Animal Care and Use 
Committee at the China Medical University. All the methods were carried out in accordance with the approved guidelines.

\section{Consent for publication}

Not applicable.

\section{Competing interests}

The authors declare that they have no competing interests.

\section{References}

1. Hardy J, Selkoe DJ. The amyloid hypothesis of Alzheimer's disease: progress and problems on the road to therapeutics. Science 2002;297:353-356

2. Sevigny J, Chiao P, Bussière T, Weinreb $P H$, Williams $L$, Maier $M$, et al. The antibody aducanumab reduces $A \beta$ plaques in Alzheimer's disease. Nature. 2016;537:50-6

3. Godyń, J. Jończyk, D. Panek, B. Malawska, Therapeutic strategies for Alzheimer's disease in clinical trials, Pharmacol. Rep.2016;68:127-138

4. Martinez B, Peplow PV, et al.Amelioration of Alzheimer's disease pathology and cognitive deficits by immunodulatory agents in animal models of Alzheimer's disease.Neural Regen Res. 2019;14:11581176

5. Holmes C, Boche D, Wilkinson D, Yadegarfar G, Hopkins V, Bayer A, Jones RW, Bullock R, Love S, Neal JW, Zotova E, Nicoll JAR: Long term effects of A 42 immunization in Alzheimer's disease: immune response, plaque removal and clinical function. Lancet 2008,372:216-223

6. Francesco Panza,Madia Lozupone,Davide Seripa,et al.Amyloid- $\beta$ immunotherapy for Alzheimer disease:Is it now a long shot?Ann 2019,85(3):303-315

7. Palmeri A, Ricciarelli R, Gulisano W, et al. Amyloid- $\beta$ peptide is needed for cGMP-induced long-term potentiation and memory. J Neurosci 2017;37:6926-6937.

8. Nisbet RM,Polanco JC,Ittner LM, Götz J.Tau aggregation and its interplay with amyloid- $\beta$. Acta Neuropathol.2015;129(2):207-20

9. Mairet-Coello G, Courchet J, Pieraut S, Courchet V, Maximov A, Polleux F.The CAMKK2-AMPK kinase pathway mediates the synaptotoxic effects of Abeta oligomers through Tau phosphorylation. Neuron.2013;78(1):94-108

10. Oddo S, Vasilevko V, Caccamo A, Kitazawa M, Cribbs DH, LaFerla FM.Reduction of soluble Abeta and tau, but not soluble Abeta alone, ameliorates cognitive decline in transgenic mice with plaques and tangles. J Biol Chem.2006; 281(51):39413-39423

11. Fa M, Staniszewski A, Saeed F, Francis YI, Arancio O. Dynamin 1 is required for memory formation. PLoS One.2014; $9:$ e91954 
12. Yu YZ,Li QL,Wang HC,et al.Improved synaptic and cognitive function in aged 3×Tg-AD mice with reduced amyloid- $\beta$ after immunotherapy with a novel recombinant 6 A $\beta 15-T F$ chimeric vaccine.Clin Immunol.2018;193:12-23

13. Mastrangelo, M.A. \& W.J. Bowers. 2008. Detailed immunohistochemical characterization of temporal and spatial progression of Alzheimer's disease related pathologies in male triple-transgenic mice. BCM Neurosci. 9: 81.

14. Schommer NN,Nguyen J,Yung BS,et al. Active immunoprophylaxis and vaccine augmentations mediated by a novel plasmid DNA formulation.Hum Gene Ther.2019;30(4):523-533

15. HY Zhang, $K$ Zhu, Y Meng,et al.Reduction of amyloid beta by Aß3-10-KLH vaccine also decreases tau pathology in 3×Tg-AD mice.Brain Res Bull.2018;142:233-240

16. Dahlgren, K.N., Manelli, A.M., Stine, W.B., Baker, L.K., Krafft, G.A., LaDu, M.J.Oligomeric and fifibrillar species of amyloid-beta peptides differentially affect neuronal viability. J. Biol. Chem. 2002;277, 32046-32053.

17. Fukuchi, K., Pham, D., Hart, M., Li, L., Lindsey, J.R. Amyloid-beta deposition in skeletal muscle of transgenic mice: possible model of inclusion body myopathy. Am. J. Pathol.1998;153:1687-1693.

18. Wang CY, Wang T, Zheng W et al (2010) Zinc overload enhances APP cleavage and Ab deposition in the Alzheimer mouse brain. PLoS ONE 5:e15349

19. Rajamohamedsait, H. B. \& Sigurdsson, E. M. Histological Staining of Amyloid and Pre-amyloid Peptides and Proteins in Mouse Tissue. Methods Mol Biol.2012;849, 411-424

20. Asuni, A. A. et al. Vaccination of Alzheimer's model mice with $A \beta$ derivative in alum adjuvant reduces $A \beta$ burden without microhemorrhages. Eur. J Neurosci.2006; 24, 2530-2542

21. Sha $S, X N$ Xing,YP Cao.Active immunotherapy facilitates $A \beta$ plaque removal following through microglial activation without obvious T cells infiltrating the CNS.Journal of Neuroimmunology.2014;274(1-2):62-70

22. Fa M, Staniszewski A, Saeed F, Francis YI, Arancio O. Dynamin 1 is required for memory formation. PLoS One.2014;9:e91954

23. C. Sinjoanu, S. Kleinschmidt, R.S. Bitner, J.D. Brioni, A. Moeller, A. Ferreira, The novel calpain inhibitor A-705253 potently inhibits oligomeric beta-amyloid-induced dynamin 1 and tau cleavage in hippocampal neurons, Neurochem. Int.2008; 53;79-88

24. Wilcock DM, Rojiani A, Rosenthal A, Subbarao S, Freeman MJ, Gordon MN, Morgan D. Passive immunotherapy against Abeta in aged APP-transgenic mice reverses cognitive deficits and depletes parenchymal amyloid deposits in spite of increased vascular amyloid and microhemorrhage. $J$ Neuroinflammation.2004;a;1:24

25. Wisniewski T, Goni F. Immunotherapeutic approaches for Alzheimer's disease. Neuron. 2015;85:1162-76

26. Lannfelt L, Relkin NR, Siemers ER. Amyloid-ß-directed immunotherapy for Alzheimer's disease. J Intern Med. 2014;275:284-95 
27. Hayk Davtyan,Armine Hovakimyan,Sepideh Kiani Shabestari,et al. Testing a MultiTEP-based combination vaccine to reduce $A \beta$ and tau pathology in Tau22/5xFAD bigenic mice.Alzheimers Res Ther.2019;11:107

28. Schelle J, Wegenast-Braun BM, Fritschi SK,et al.Early A $\beta$ Reduction Prevents Progression of Cerebral Amyloid Angiopathy.Ann Neurol.2019;86(4):561-571

29. Mairet-Coello G, Courchet J, Pieraut S, et al.The CAMKK2-AMPK kinase pathway mediates the synaptotoxic effects of Abeta oligomers through Tau phosphorylation. Neuron.2013;78(1):94-108

30. Yu W, Polepalli J, Wagh D, Rajadas J, Malenka R, Lu B.A critical role for the PAR-1/MARK-tau axis in mediating the toxic effects of Abeta on synapses and dendritic spines. Hum Mol Genet.2012;21(6):1384-1390

31. Karran E, Hardy J. Antiamyloid therapy for Alzheimer's disease-are we on the right road? N Engl J Med. 2014; 370(4):377-8

32. Das, P., Murphy, M.P., Younkin, L.H., Younkin, S.G., and Golde, T.E. Reduced effectiveness of Abeta1-42 immunization in APP transgenic mice with signifificant amyloid deposition. Neurobiol. Aging.2001; 22, 721-727

33. Irina Petrushina, Hayk Davtyan,Armine Hovakimyan,et al.Comparison of efficacy of preventive and therapeutic vaccine targeting the $\mathrm{N}$ terminus of $\beta$-amyloid in an animal model of Alzheimer's Disease.Mol Ther.2017;25(1):153-164

34. Alex Allen,Chuan Wang,Lisa J Caproni, et al.Linear Doggybone DNA vaccine induces similar immunological responses to conventional plasmid DNA independently of immune recognition by TLR9 in a pre-clinical model.Cancer Immunol Immunother.2018;67(4):627-38

35. Yu YZ, Wang WB, Chao A, Chang Q, Liu S, Zhao M, et al. Strikingly reduced amyloid burden and improved behavioral performance in Alzheimer's disease mice immunized with recombinant chimeric vaccines by hexavalent foldable A $1-15$ fused to toxin-derived carrier proteins. J Alzheimers Dis 2014;41:243-60

36. Nussbaum, J. M., Schilling, S., Cynis, H., et al.Prion like behaviour and tau-dependent cytotoxicity of pyroglutamylated amyloid. Nature.2012;485, 651-655

37. Rangaraju S, Dammer EB, Raza SA,et al.Quantitative Proteomics of Acutely-Isolated Mouse Microglia Identifies Novel Immune Alzheimer's Disease-Related Proteins.Mol Neurodegener.2018;13(1):34

38. Zotova, C. Holmes, D. Johnston,et al.Microglial alterations in human Alzheimer's disease following Aß42 immunization. Neuropathology and Applied Neurobiology.2011;37:513-524

39. Braak H, Alafuzoff I, Arzberger T, Kretzschmar H, Del Tredici K. Staging of Alzheimer disease associated neurofibrillary pathology using paraffin sections and immunocytochemistry. Acta Neuropathol 2006;112:389-404

40. Pradeepkiran JA,Reddy PH.Structure based desing and molecular docking studies for phosphorylated tau inhibitors in Alzheimer's disease.Cells.2019;8(3):260

41. Xia Z,Wang F,Zhou S,et al.Catapol protects synaptic proteins from beta-amyloid induced neuron injury and improves cognitive functions in aged rats.Oncotarget.2017;8(41):69303-15 
Figures

A

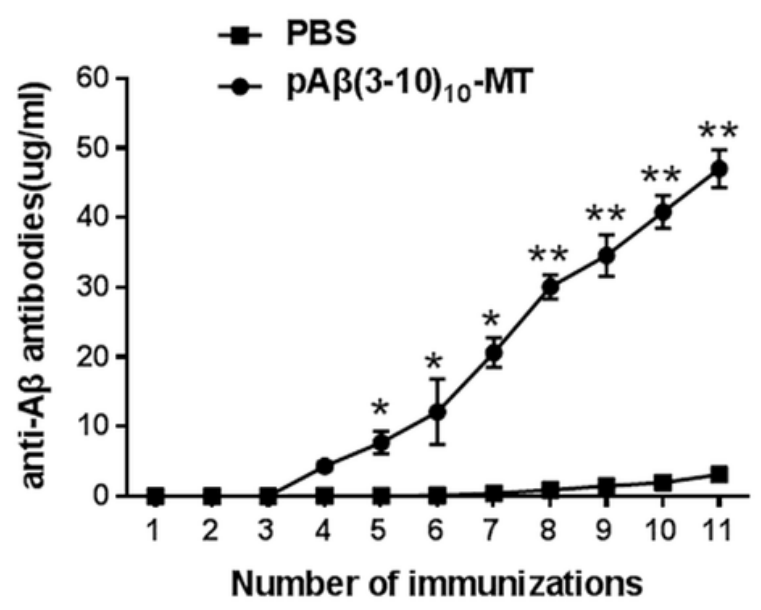

B

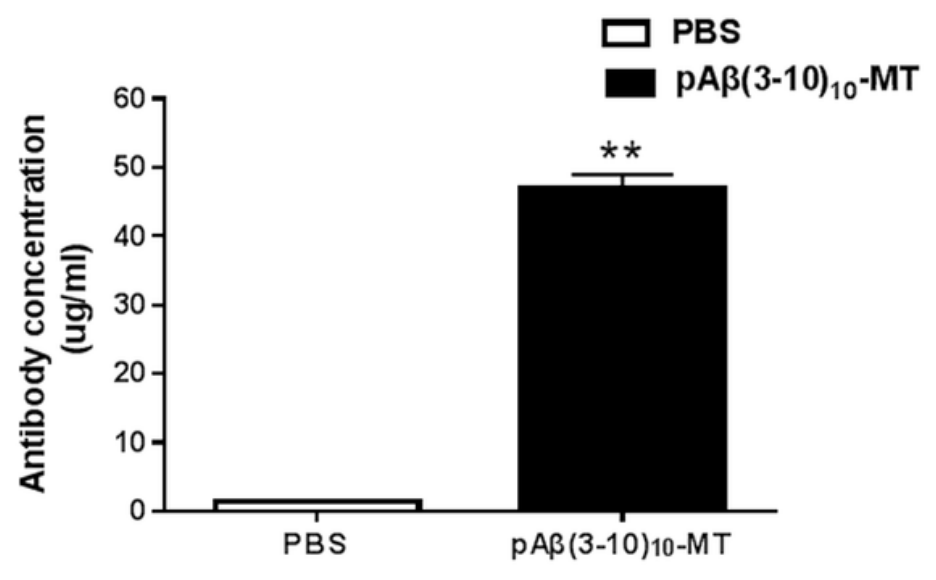

$\mathrm{C}$

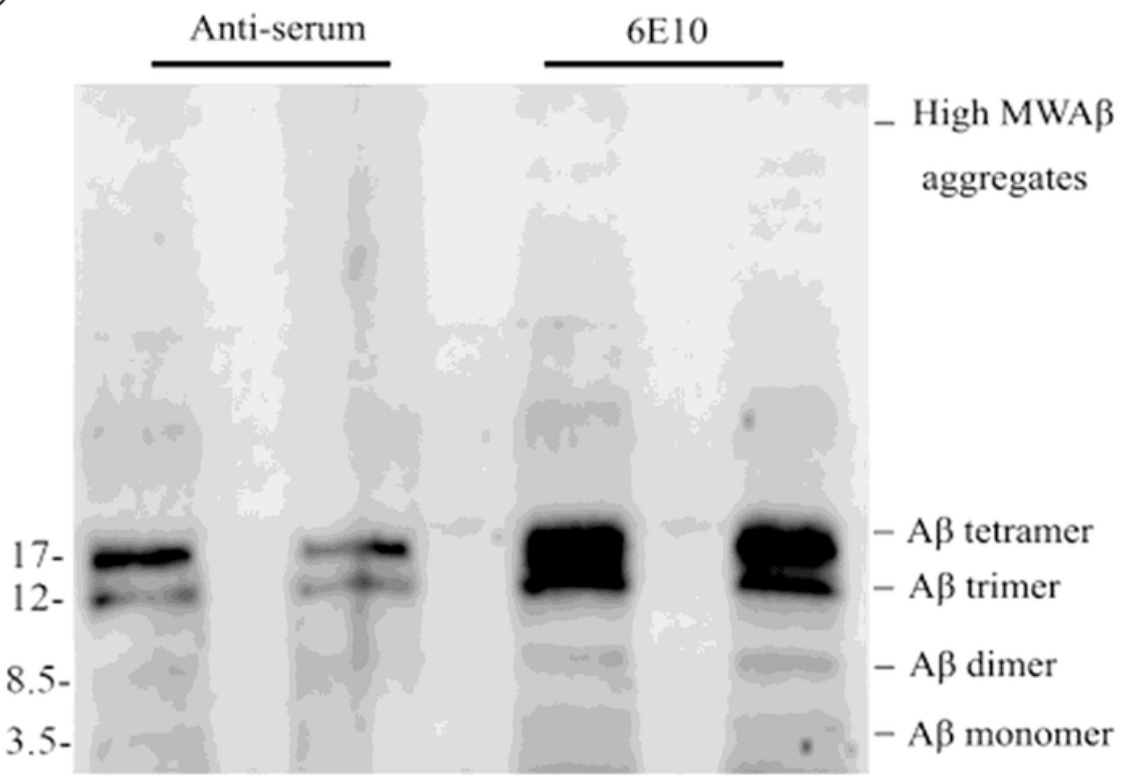

Figure 1

Levels and immunogenicity of the anti-A $\beta$ antibody.(A).The anti-A $\beta$ antibodies can be detected in $p(A \beta 3-$ 10)10-MT immunized 3×Tg-AD mice while no antibody titer be discovered in the PBS group mice. (B).The antibody exhibited obvious higher concentration in $\mathrm{p}(\mathrm{A} \beta 3-10) 10 \mathrm{MT}$ immunized group other than the PBS group. $P<0.01$.(C).Antibody of $p(A B 3-10) 10-M T$ immunized serum binds strongly to $A \beta 42$ oligomers, but minimally to $A \beta 42$ fibrils. 
A

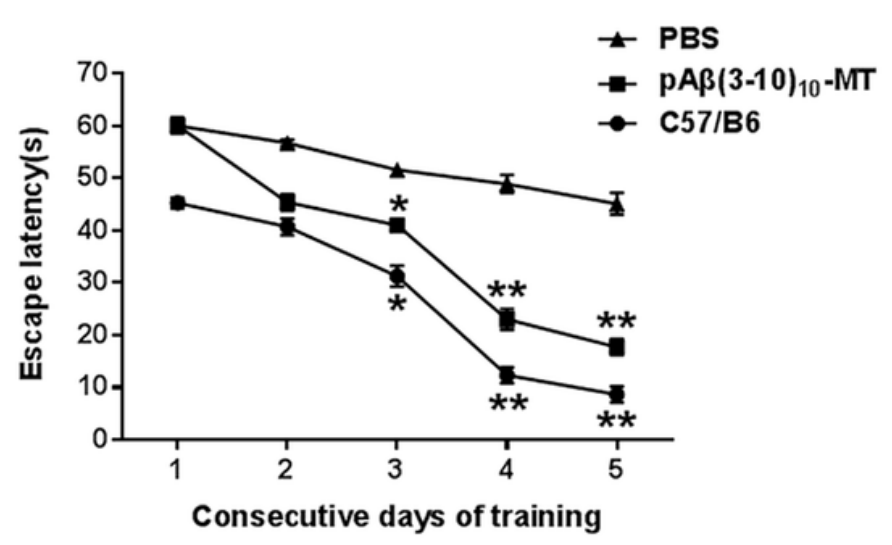

B

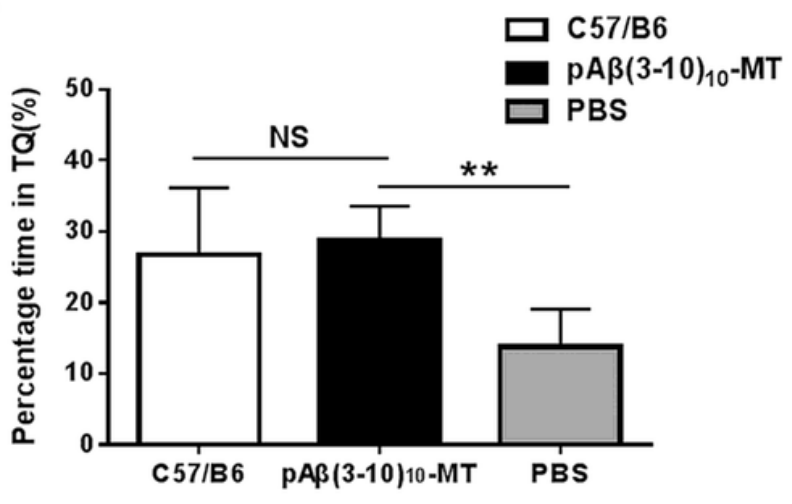

$\mathrm{C}$

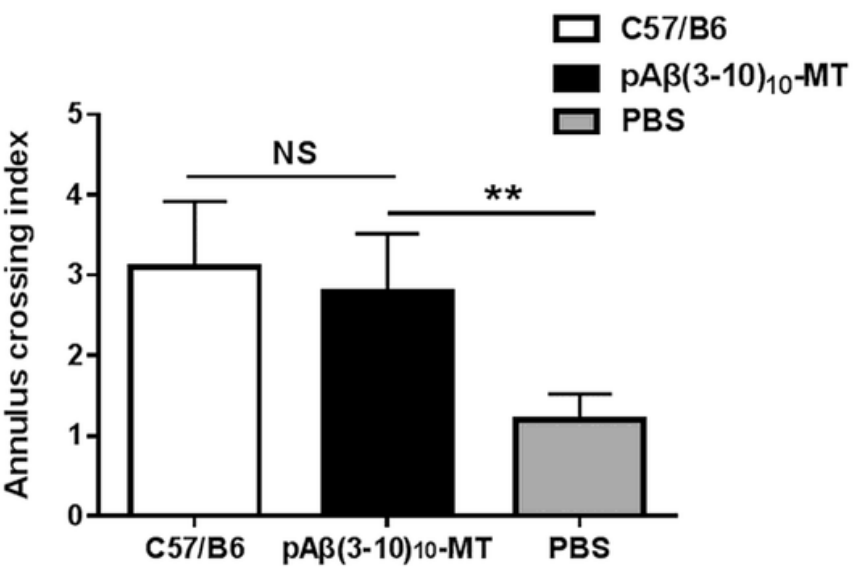

Figure 2

Morris water maze test to evaluate the cognitive function.(A).The escape latency was significantly reduced for $p(A \beta 3-10) 10-M T$-immunized group than the PBS group. $P<0.01$.(B). In probe test, initial incubation latency in $p(A B 3-10) 10-M T$ group was significantly shortened, $p<0.01$.(C). Mice in $p(A \beta 3-$ 10)10-MT group cross the correct platform location more often than the control group, $p<0.01$. There was no difference between the performance of the $p(A \beta 3-10) 10-M T$ group and the wild-type group mice.PQ 0.05 
A

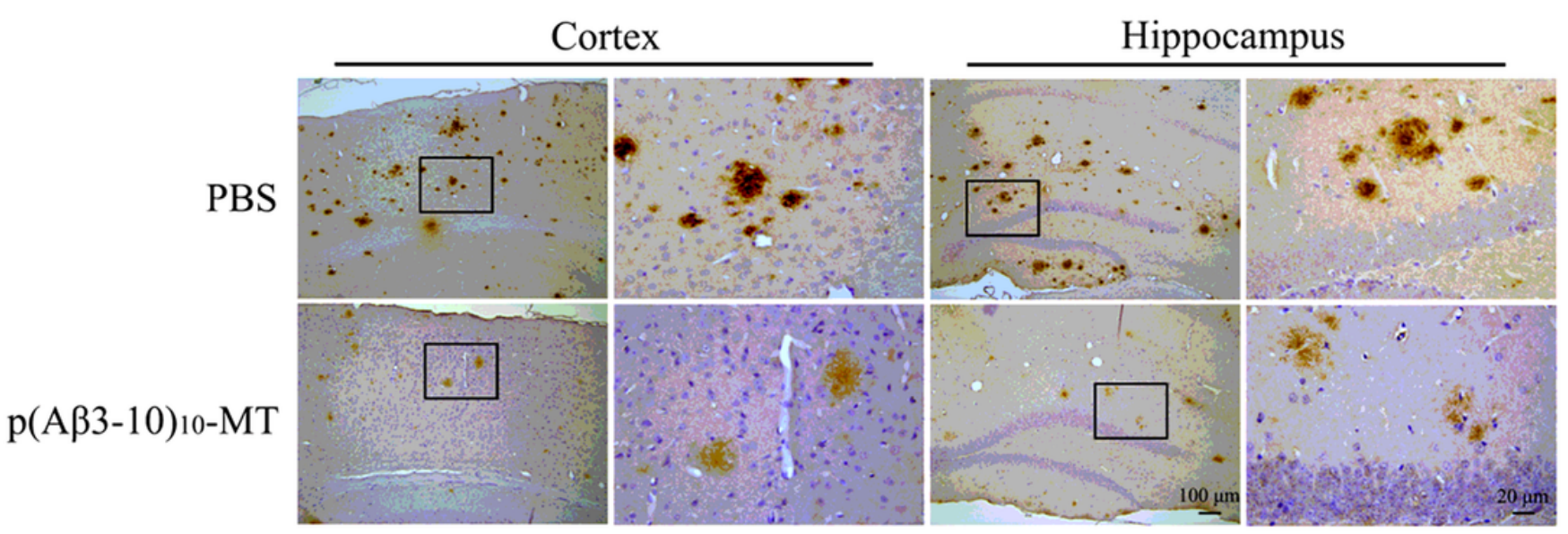

B

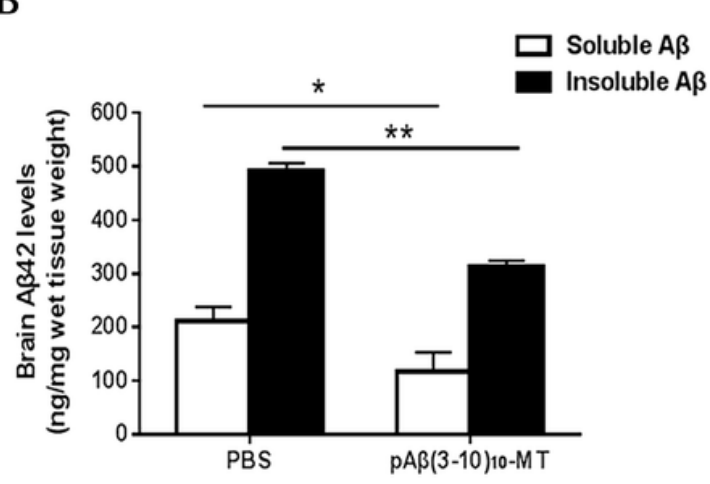

C

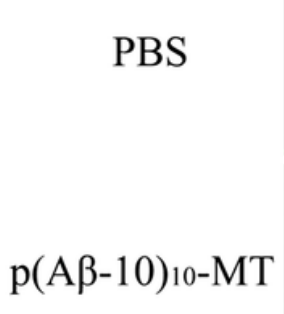

$\mathrm{A} \beta$
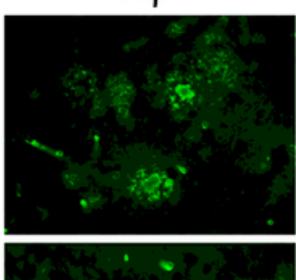

IBA-1

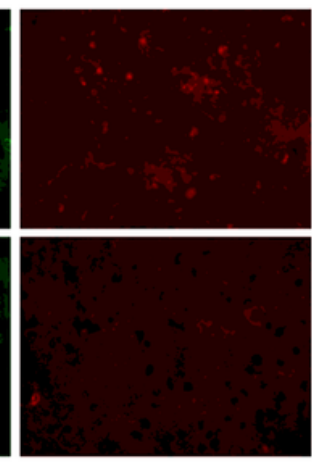

Merge

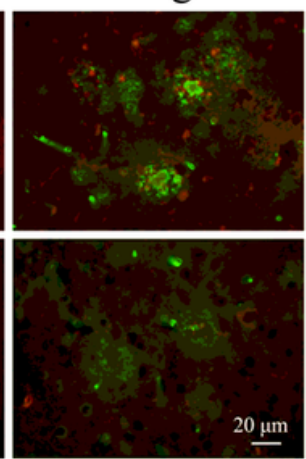

\section{Figure 3}

The amyloid burden were reduced through microglia activation.(A).Amyloid burden were reduced in the both cortex and hippocampus for the $\mathrm{p}(\mathrm{A} \beta 3-10) 10-\mathrm{MT}$ immunized mice compared with PBS-immunized group. $P<0.05$. (B).The levels of soluble and insoluble $A \beta 42$ peptide were decreased compared to unimmunized mice.P< 0.05.(C). More lba-1 positive cells clustered around senile plaques in the brain of $\mathrm{p}(\mathrm{A \beta 3}-10)$ 10-MT immunized mice. 
A

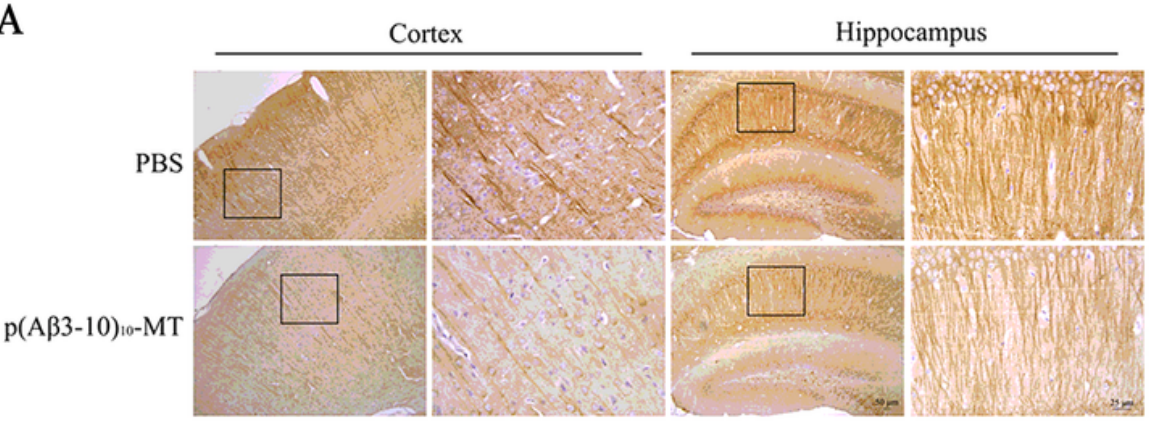

$\mathrm{C}$

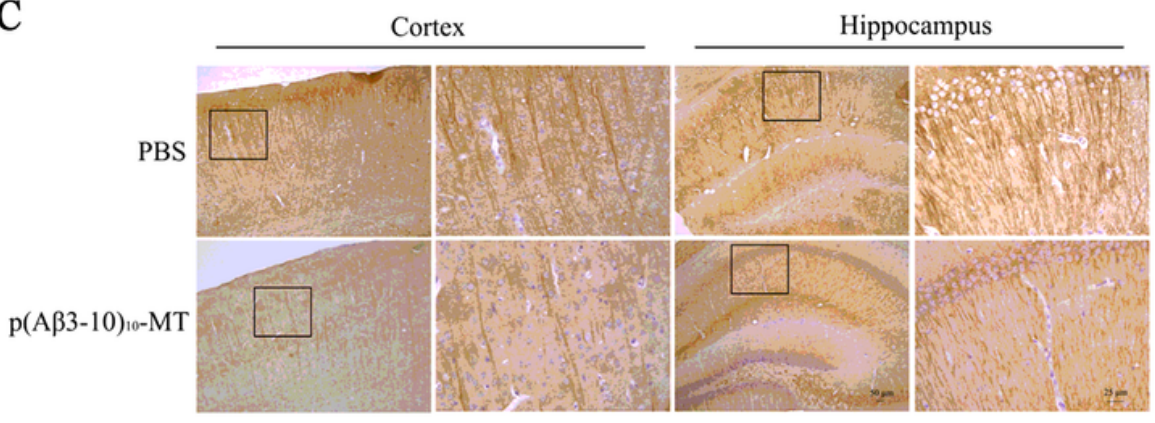

$\mathrm{B}$

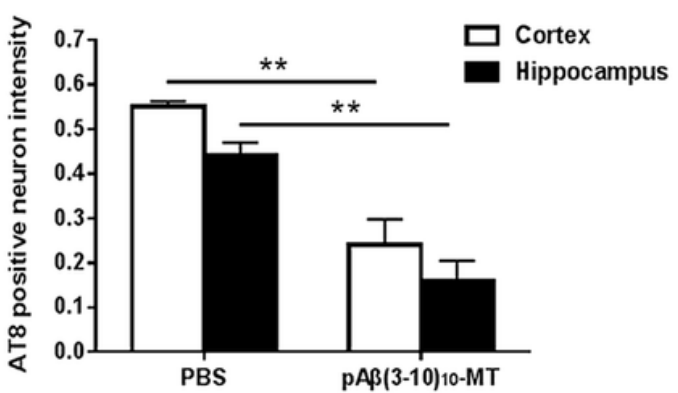

$\mathrm{D}$

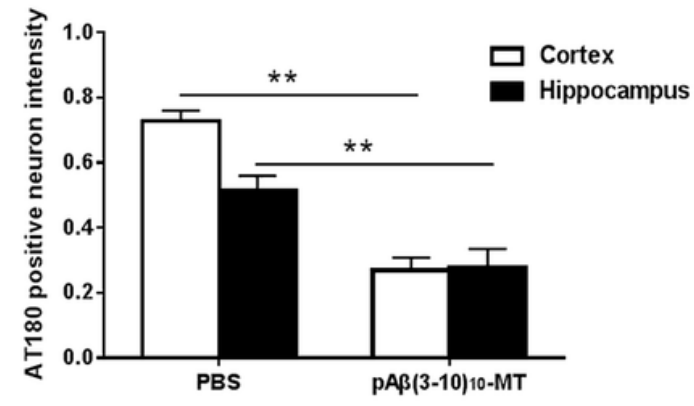

$\mathrm{E}$

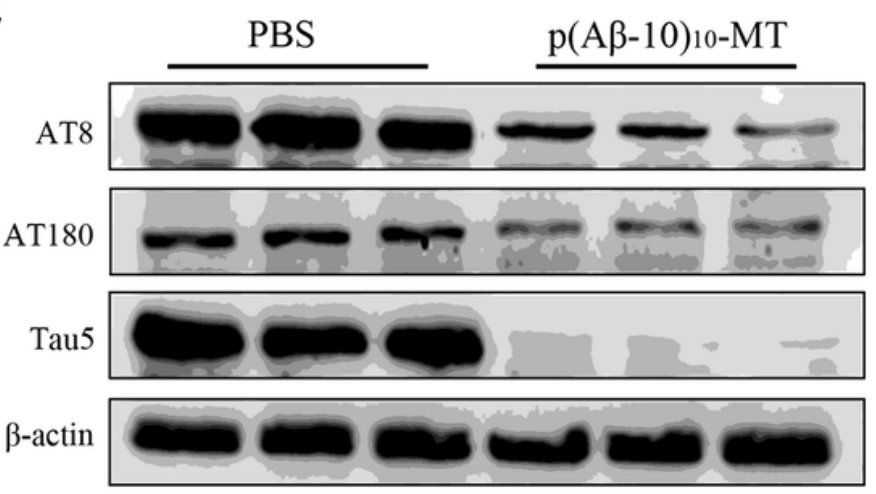

Figure 4

Detection of phosphorylated tau protein level.(A,B).AT8-positive neurons were reduced after vaccination. $P<0.05$. (C,D).AT180-positive neurons were reduced after vaccination. $P<0.05$. (E).Western blot to detect AT8, AT180 and total tau levels. 
A
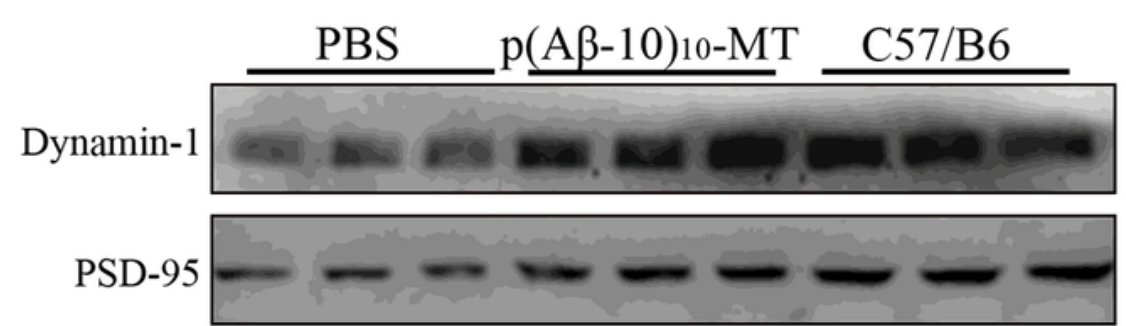

Synapsin-1
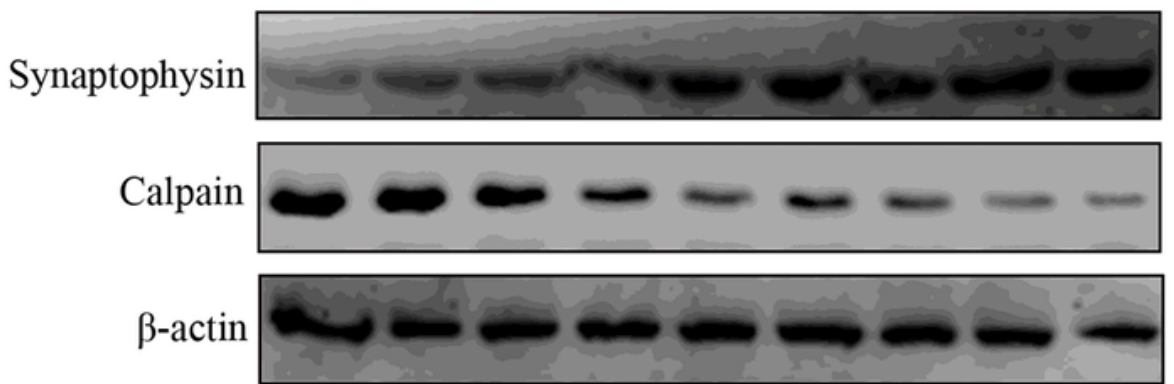

B

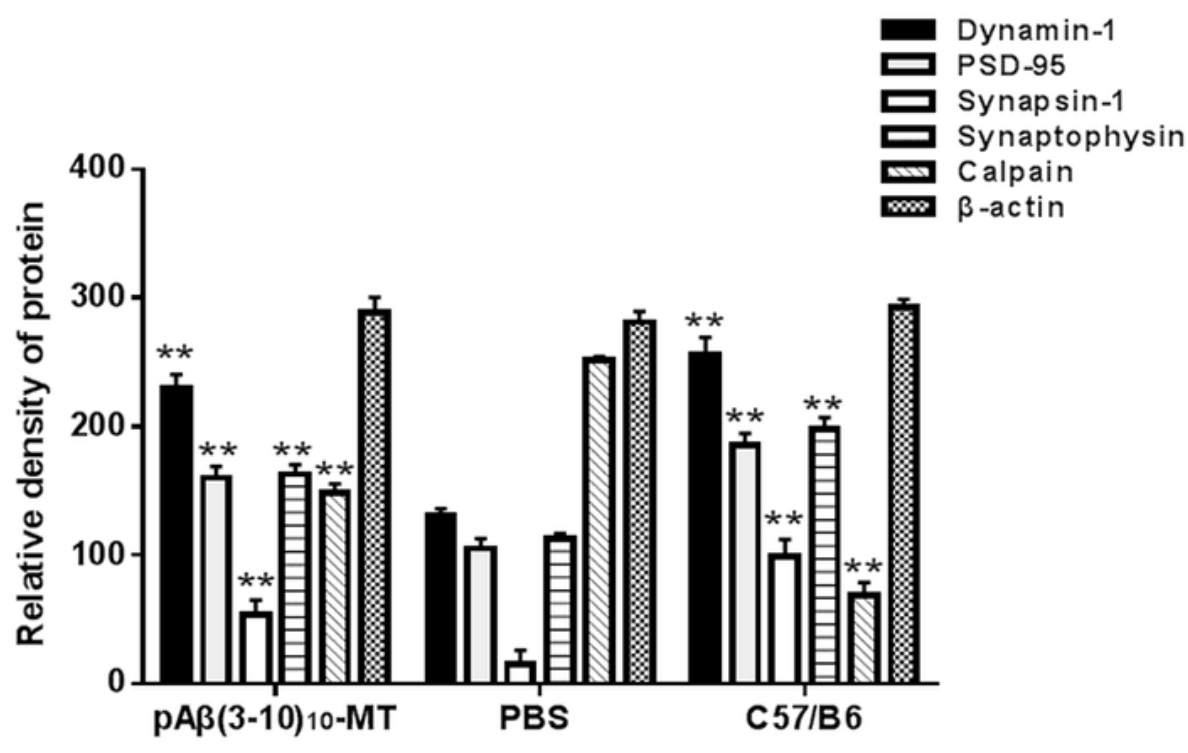

\section{Figure 5}

Analysis of synaptic protein to evaluate synaptic function. Dynamin 1, PSD-95, synaptophysin and synapsin I were reduce in 3×Tg-AD mice while $\mathrm{p}(\mathrm{A} \beta 3-10) 10-\mathrm{MT}$ immunization can prevent this decline. The opposite tendency was seen in calpain. $P<0.01$. 
A
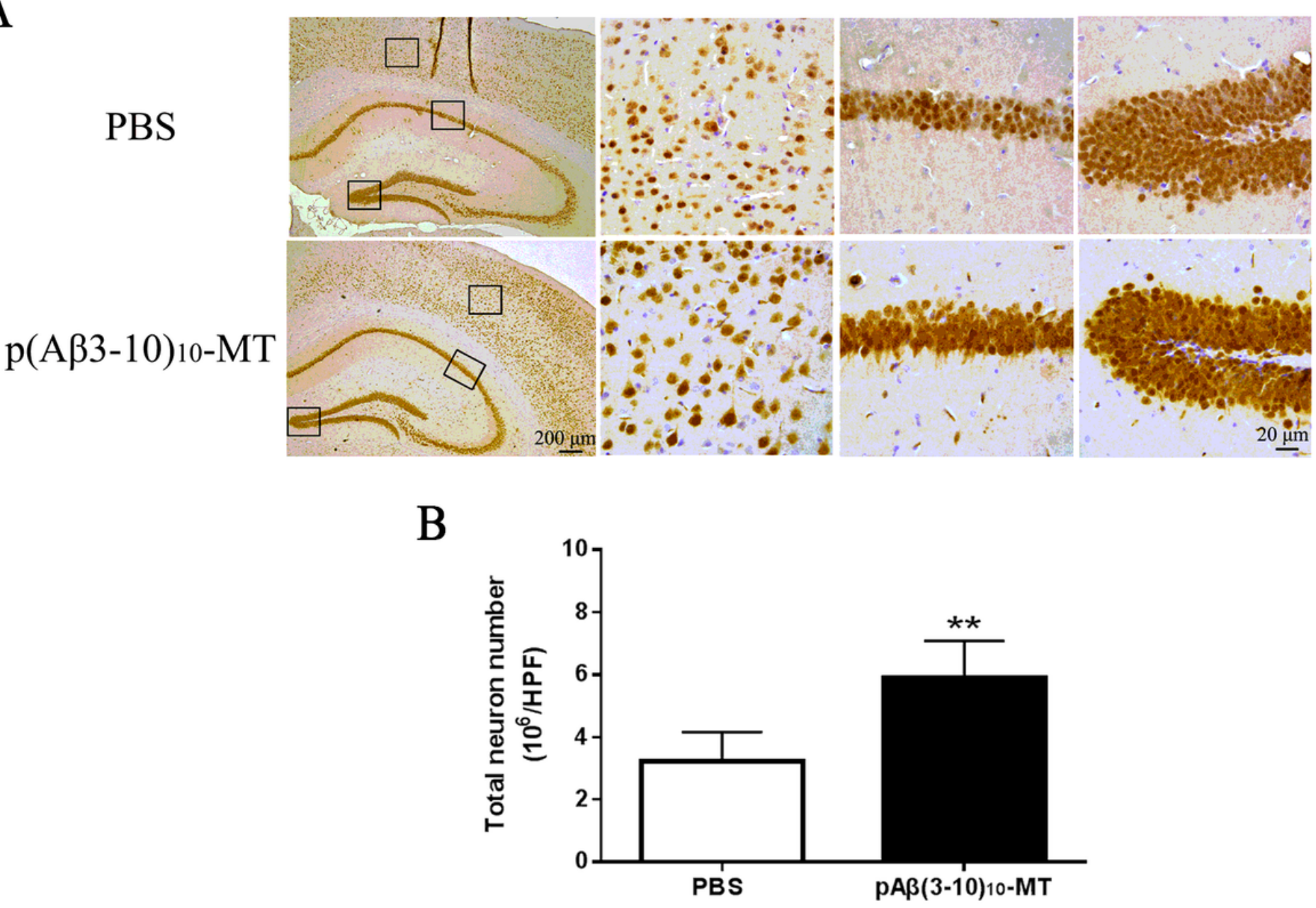

Figure 6

NeuN immunohistochemistry shows loss of neuron can be prevented after immunization. It shows a $46 \%$ loss in $3 \times \mathrm{Tg}-\mathrm{AD}$ mice immunized with PBS while only $11 \%$ of neurons in mice immunized with $\mathrm{p}(\mathrm{A \beta} 3-$ 10)10-MT was lost.P $<0.01$.

A

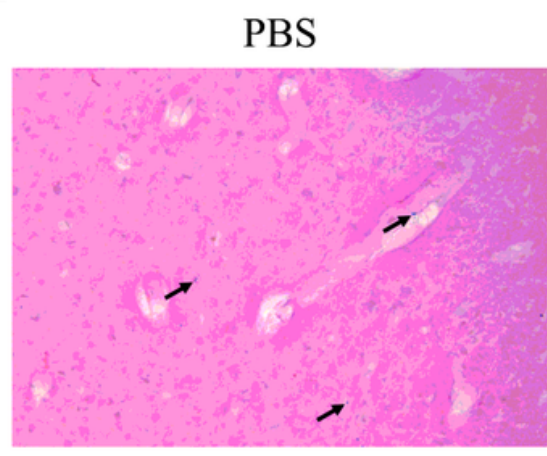

$\mathrm{p}(\mathrm{A} \beta-10) 10-\mathrm{MT}$

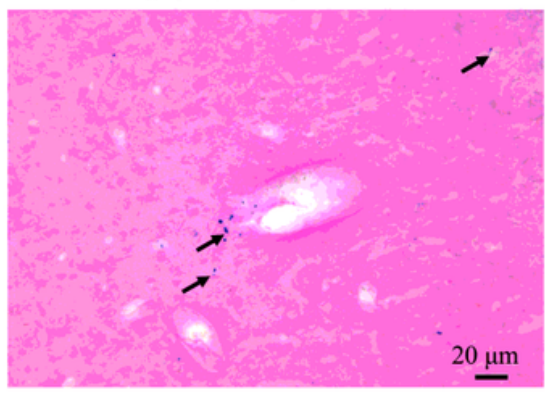

B

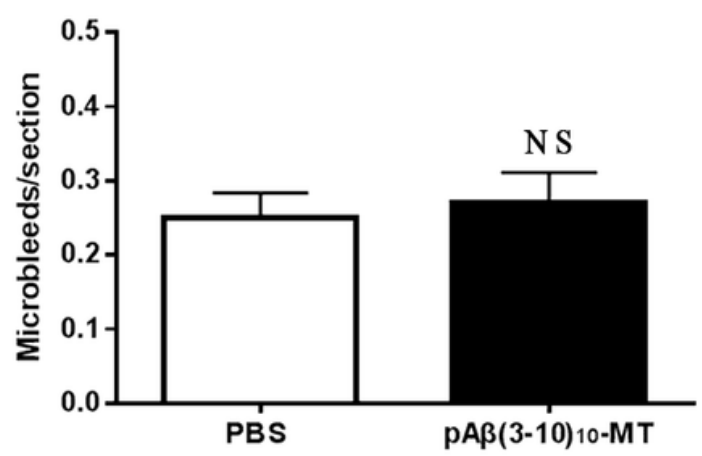

Figure 7 
Prussian blue histological staining represent there was no obvious microhemorrhage in $p(A \beta 3-10) 10-M T$ immunized transgenic mice. 